\title{
On a conjecture of Widom
}

\author{
Alexei Borodin $^{1}$ and Alexei Novikov ${ }^{2}$ \\ ${ }^{1}$ Department of Mathematics, California Institute of Technology, Mathematics 253-37, Caltech, \\ Pasadena, CA 91125, USA \\ ${ }^{2}$ Department of Mathematics, Penn State University, University Park, State College, PA 16802, \\ USA
}

Received 9 September 2005, in final form 14 November 2005

Published 27 June 2006

Online at stacks.iop.org/JPhysA/39/8895

\begin{abstract}
We prove a conjecture of Widom (2002 Int. Math. Res. Not. 455-64 (Preprint math/0108008)) about the reality of eigenvalues of certain infinite matrices arising in asymptotic analysis of large Toeplitz determinants. As a byproduct, we obtain a new proof of Okounkov's formula for the (determinantal) correlation functions of the Schur measures on partitions.
\end{abstract}

PACS numbers: $02.10 . \mathrm{Eb}, 05.40 .+\mathrm{j}$

\section{Introduction}

Consider an operator $T$ in $L^{2}(n, n+1, \ldots), n=0,1, \ldots$, with matrix elements

$$
T_{p q}=\sum_{k \geqslant 1}\left(\frac{\phi_{-}}{\phi_{+}}\right)_{p+k}\left(\frac{\phi_{+}}{\phi_{-}}\right)_{-q-k}
$$

where $(f)_{k}$ denotes the $k$ th Fourier coefficient of a function $f(z)$ on the unit circle $\{z \in \mathbb{C}:|z|=1\}$ and the functions

$$
\phi_{+}(z)=\mathrm{e}^{\gamma^{+} z} \prod_{i \geqslant 1} \frac{1+\beta_{i}^{+} z}{1-\alpha_{i}^{+} z}, \quad \phi_{-}(z)=\mathrm{e}^{\gamma^{-} / z} \prod_{i \geqslant 1} \frac{1+\beta_{i}^{-} / z}{1-\alpha_{i}^{-} / z}
$$

are determined by certain nonnegative parameters $\left\{\alpha_{i}^{ \pm}\right\},\left\{\beta_{i}^{ \pm}\right\}$and $\gamma^{ \pm}$such that $\sum_{i}\left(\alpha_{i}^{ \pm}+\right.$ $\left.\beta_{i}^{ \pm}\right)<\infty$ and $\alpha_{i}^{ \pm}, \beta_{i}^{ \pm} \leqslant$const $<1$ for all $i \geqslant 1$. The main goal of this paper is to prove that the spectrum of this operator is real and lies between 0 and 1 .

This property of the operator $T$ turns out to be useful in the asymptotic analysis of growing Toeplitz determinants with symbol $\phi=\phi_{+} \phi_{-}$or, more generally, of the distribution functions of the largest parts of random partitions distributed according to the associated Schur measure. The statement was conjectured by Widom in [W] in the case when the parameters $\alpha_{i}^{+}, \beta_{i}^{-}, \gamma^{ \pm}$ vanish and the number of nonzero $\alpha_{i}^{-} \mathrm{s}$ and $\beta_{i}^{+} \mathrm{s}$ is finite. Some more special cases were proved in $[\mathrm{BDR}, \mathrm{W}]$. We refer to $[\mathrm{W}]$ for details and further references. 
The key fact which allows us to prove the reality of the spectrum is that the resolvent $T(1-T)^{-1}$ of matrix $T$ after conjugation by a diagonal matrix with plus-minus 1 s becomes totally positive. While proving this fact, we obtain as a byproduct a new proof of Okounkov's formula for the correlation functions of the Schur measures, see [O] and also [J, R] for other proofs. More specifically, we show that the Schur measure may be viewed as an $L$-ensemble and explicitly compute the correlation kernel $K=L(1+L)^{-1}$.

\section{An operator identity}

In what follows, $\mathbb{Z}_{+}$denotes the set of nonnegative integers and all contour integrals are taken over circles centred at the origin with radii close enough to 1 .

Let $\phi_{+}$be a holomorphic function in the disc

$$
D_{r}=\{z \in \mathbb{C}:|z| \leqslant r\}
$$

with radius $r>1$ and let $\phi_{-}$be a holomorphic function outside the disc $D_{1 / r}$. In other words,

$$
\phi_{ \pm}(z)=\sum_{n=0}^{\infty}\left(\phi_{ \pm}\right)_{n} z^{ \pm n}, \quad\left(\phi_{ \pm}\right)_{n}=O\left(r^{-n}\right) \quad \text { as } \quad n \rightarrow \infty .
$$

Also assume that $\phi_{+}$and $\phi_{-}$do not vanish on $D_{r}$ and $\overline{\mathbb{C}} \backslash D_{1 / r}$, respectively.

Consider an operator $L$ in $L^{2}\left(\mathbb{Z}_{+}\right) \oplus L^{2}\left(\mathbb{Z}_{+}\right)$with matrix

$$
L=\left[\begin{array}{cc}
0 & A^{t} \\
-B & 0
\end{array}\right]
$$

where the generating functions of matrix elements of $A$ and $B$ are

$$
\sum_{p, q \geqslant 0} A_{p q} u^{p} v^{q}=\frac{1}{u+v}\left(\frac{\phi_{+}(u)}{\phi_{+}(-v)}-1\right), \quad \sum_{p, q \geqslant 0} B_{p q} u^{p} v^{q}=\frac{1}{u+v}\left(\frac{\phi_{-}\left(u^{-1}\right)}{\phi_{-}\left(-v^{-1}\right)}-1\right) .
$$

One can also write the matrix elements in terms of the contour integrals

$$
\begin{aligned}
& A_{p q}=\frac{1}{(2 \pi \mathrm{i})^{2}} \oint \oint\left(\frac{\phi_{+}(u)}{\phi_{+}(-v)}-1\right) \frac{\mathrm{d} u \mathrm{~d} v}{(u+v) u^{p+1} v^{q+1}}, \\
& B_{p q}=\frac{1}{(2 \pi \mathrm{i})^{2}} \oint \oint\left(\frac{\phi_{-}\left(u^{-1}\right)}{\phi_{-}\left(-v^{-1}\right)}-1\right) \frac{\mathrm{d} u \mathrm{~d} v}{(u+v) u^{p+1} v^{q+1}} .
\end{aligned}
$$

These integral representations imply that $\left|A_{p q}\right|$ and $\left|B_{p q}\right|$ decay faster than const $\cdot x^{-p-q}$ for any $1<x<r$ as $p+q \rightarrow \infty$. Thus, the sum of absolute values of matrix elements of $L$ is finite, and $L$ is a trace class operator.

Note that the change $\phi_{+}(z) \leftrightarrow \phi_{+}^{-1}(-z)$ replaces $A$ by $A^{t}$, the change $\phi_{-}(z) \leftrightarrow \phi_{-}^{-1}(-z)$ replaces $B$ by $B^{t}$ and the change

$$
\left(\phi_{+}(u), \phi_{-}(v)\right) \longleftrightarrow\left(\phi_{-}\left(u^{-1}\right), \phi_{+}\left(v^{-1}\right)\right)
$$

switches $A$ and $B$. Thus, the switch $(A, B) \leftrightarrow\left(B^{t}, A^{t}\right)$ is achieved by

$$
\left(\phi_{+}(u), \phi_{-}(v)\right) \longleftrightarrow\left(\phi_{-}^{-1}\left(-u^{-1}\right), \phi_{+}^{-1}\left(-v^{-1}\right)\right) .
$$

Theorem 1. Assume that the operator $1+A^{t} B$ is invertible (equivalently, $1+B A^{t} i$ s invertible). Then the operator $1+L$ is invertible, and the matrix of the operator $K=$ $L(1+L)^{-1}=1-(1+L)^{-1}$ has the form

$$
K=\left[\begin{array}{ll}
K_{11} & K_{12} \\
K_{21} & K_{22}
\end{array}\right]=\left[\begin{array}{cc}
1-\left(1+A^{t} B\right)^{-1} & \left(1+A^{t} B\right)^{-1} A^{t} \\
-\left(1+B A^{t}\right)^{-1} B & 1-\left(1+B A^{t}\right)^{-1}
\end{array}\right]
$$


where

$$
\begin{aligned}
& \left(K_{11}\right)_{p q}=\delta_{p q}-\frac{(-1)^{p+q}}{(2 \pi \mathrm{i})^{2}} \oint \oint_{|z w|<1} \frac{\Phi(z, w) \mathrm{d} z \mathrm{~d} w}{(1-z w) z^{p+1} w^{q+1}}, \\
& \left(K_{12}\right)_{p q}=\frac{(-1)^{p}}{(2 \pi \mathrm{i})^{2}} \oint \oint_{|z w|<1} \frac{\Phi(z, w) w^{q} \mathrm{~d} z \mathrm{~d} w}{(1-z w) z^{p+1}}, \\
& \left(K_{21}\right)_{p q}=\frac{(-1)^{q+1}}{(2 \pi \mathrm{i})^{2}} \oint \oint_{|z w|<1} \frac{\Phi(z, w) w^{p} \mathrm{~d} z \mathrm{~d} w}{(1-z w) z^{q+1}}, \\
& \left(K_{22}\right)_{p q}=\frac{1}{(2 \pi \mathrm{i})^{2}} \oint \oint_{|z w|<1} \frac{\Phi(z, w) z^{p} w^{q} \mathrm{~d} z \mathrm{~d} w}{1-z w},
\end{aligned}
$$

and

$$
\Phi(z, w)=\frac{\phi_{-}(z) \phi_{+}\left(w^{-1}\right)}{\phi_{+}(z) \phi_{-}\left(w^{-1}\right)}
$$

Comment. The proof of this statement given below is a verification of the relation $(1-K)(1+L)=1$ rather than the derivation of the inverse of the operator $1+L$, so the reader might wonder where the formulae for the operator K above came from. The answer to this question is hidden in theorem 3.

Symmetric minors of the matrix $L$ are the weights of partitions with respect to the socalled Schur measure. Simple linear algebra implies that, consequently, minors of the matrix $K=L(1+L)^{-1}$ must be the correlation functions of the Schur measure. Another matrix $\widetilde{K}$ whose minors give these correlation functions has been previously computed in [O], and the formulae for $K$ above were obtained from the known formula for $\widetilde{K}$ by conjugation by $a$ diagonal matrix with plus-minus 1 s on the diagonal. Some details can be found in the next section and references therein.

Proof. The proof of the first equality is straightforward. Thanks to the symmetries mentioned before the statement of the theorem it suffices to prove the integral formulae for $K_{11}$ and $K_{12}$. Let us start with $K_{11}$.

We need to show that $\left(1+A^{t} B\right)\left(1-K_{11}\right)=1$ or, equivalently, $A^{t} B\left(1-K_{11}\right)=K_{11}$. Explicit computation gives

$$
\begin{aligned}
\left(A^{t} B\left(1-K_{11}\right)\right)_{p q}= & \sum_{l, m \geqslant 0} A_{l p} B_{l m}\left(1-K_{11}\right)_{m q}=\frac{1}{(2 \pi \mathrm{i})^{6}} \oint \cdots \oint_{|z w|<1} \sum_{l, m \geqslant 0} \\
& \times\left(\frac{\phi_{+}\left(u_{1}\right)}{\phi_{+}\left(-v_{1}\right)}-1\right) \frac{\mathrm{d} u_{1} \mathrm{~d} v_{1}}{\left(u_{1}+v_{1}\right) u_{1}^{l+1} v_{1}^{p+1}}\left(\frac{\phi_{-}\left(u_{2}^{-1}\right)}{\phi_{-}\left(-v_{2}^{-1}\right)}-1\right) \\
& \times \frac{\mathrm{d} u_{2} \mathrm{~d} v_{2}}{\left(u_{2}+v_{2}\right) u_{2}^{l+1} v_{2}^{m+1}} \frac{\phi_{-}(z) \phi_{+}\left(w^{-1}\right)}{\phi_{+}(z) \phi_{-}\left(w^{-1}\right)} \frac{\mathrm{d} z \mathrm{~d} w}{(1-z w)(-z)^{m+1}(-w)^{q+1}} \\
= & \frac{1}{(2 \pi \mathrm{i})^{6}} \oint \cdots \oint_{|z w|<1,\left|u_{1} u_{2}\right|>1,\left|v_{2} z\right|>1}\left(\frac{\phi_{+}\left(u_{1}\right)}{\phi_{+}\left(-v_{1}\right)}-1\right) \frac{\mathrm{d} u_{1} \mathrm{~d} v_{1}}{\left(u_{1}+v_{1}\right) v_{1}^{p+1}} \\
& \times\left(\frac{\phi_{-}\left(u_{2}^{-1}\right)}{\phi_{-}\left(-v_{2}^{-1}\right)}-1\right) \frac{\mathrm{d} u_{2} \mathrm{~d} v_{2}}{\left(u_{2}+v_{2}\right)} \frac{\phi_{-}(z) \phi_{+}\left(w^{-1}\right)}{\phi_{+}(z) \phi_{-}\left(w^{-1}\right)} \\
& \times \frac{\mathrm{d} z \mathrm{~d} w}{(-w)^{q+1}} \frac{(-1)}{\left(u_{1} u_{2}-1\right)\left(v_{2} z+1\right)(1-z w)}
\end{aligned}
$$


where we imposed additional conditions $\left|u_{1} u_{2}\right|>1,\left|v_{2} z\right|>1$ on the integration contours to ensure the convergence of two geometric series under the integral.

We can immediately perform the integration over $u_{2}$ and $v_{2}$. Indeed, there is only one simple pole $u_{2}=u_{1}^{-1}$ inside the $u_{2}$-contour and there is only one simple pole $v_{2}=-z^{-1}$ inside the $v_{2}$-contour. Evaluating the residues, we obtain that the integral above equals

$$
\begin{aligned}
\frac{1}{(2 \pi \mathrm{i})^{4}} \oint \cdots & \oint_{|z w|<1}\left(\frac{\phi_{+}\left(u_{1}\right)}{\phi_{+}\left(-v_{1}\right)}-1\right) \frac{\mathrm{d} u_{1} \mathrm{~d} v_{1}}{\left(u_{1}+v_{1}\right) v_{1}^{p+1}} \\
& \quad \times\left(\frac{\phi_{-}\left(u_{1}\right)}{\phi_{-}(z)}-1\right) \frac{1}{\left(z-u_{1}\right)} \frac{\phi_{-}(z) \phi_{+}\left(w^{-1}\right)}{\phi_{+}(z) \phi_{-}\left(w^{-1}\right)} \frac{\mathrm{d} z \mathrm{~d} w}{(-w)^{q+1}} \frac{(-1)}{(1-z w)}
\end{aligned}
$$

Let us choose the contours so that $\left|u_{1}\right|<|z|$ and open the parentheses $\left(\frac{\phi_{-}\left(u_{1}\right)}{\phi_{-}(z)}-1\right)$ in the integral above. The second term vanishes because it has no singularities inside the $u_{1}$-contour. The first term has only one simple pole $z=u_{1}$ inside the $z$-contour, and the integration over $z$ gives

$\frac{1}{(2 \pi \mathrm{i})^{3}} \oint \oint \oint_{\left|u_{1} w\right|<1}\left(\frac{\phi_{+}\left(u_{1}\right)}{\phi_{+}\left(-v_{1}\right)}-1\right) \frac{\mathrm{d} u_{1} \mathrm{~d} v_{1}}{\left(u_{1}+v_{1}\right) v_{1}^{p+1}} \frac{\phi_{-}\left(u_{1}\right) \phi_{+}\left(w^{-1}\right)}{\phi_{+}\left(u_{1}\right) \phi_{-}\left(w^{-1}\right)} \frac{\mathrm{d} w}{(-w)^{q+1}} \frac{(-1)}{\left(1-u_{1} w\right)}$

Now let us choose the contours so that $\left|u_{1}\right|>\left|v_{1}\right|$ and open the parentheses $\left(\frac{\phi_{+}\left(u_{1}\right)}{\phi_{+}\left(-v_{1}\right)}-1\right)$. The first term can be integrated over $u_{1}$-there is only one pole $u_{1}=-v_{1}$ inside the $u_{1}$-contour. Thus, the first term equals the corresponding residue, that is

$$
\frac{-1}{(2 \pi \mathrm{i})^{2}} \oint \oint_{\left|v_{1} w\right|<1} \frac{\phi_{+}\left(w^{-1}\right)}{\phi_{+}\left(-v_{1}\right)} \frac{\mathrm{d} v_{1} \mathrm{~d} w}{\left(1+v_{1} w\right) v_{1}^{p+1}(-w)^{q+1}} \text {. }
$$

By deforming the $w$-contour to $\infty$ and picking up the residue at $w=-v_{1}^{-1}$, we immediately see that this integral is equal to $\delta_{p q}$.

Now the second term is equal to

$$
\frac{1}{(2 \pi \mathrm{i})^{3}} \oint \oint \oint_{\left|u_{1} w\right|<1,\left|u_{1}\right|>\left|v_{1}\right|} \frac{\mathrm{d} u_{1} \mathrm{~d} v_{1}}{\left(u_{1}+v_{1}\right) v_{1}^{p+1}} \frac{\phi_{-}\left(u_{1}\right) \phi_{+}\left(w^{-1}\right)}{\phi_{+}\left(u_{1}\right) \phi_{-}\left(w^{-1}\right)} \frac{\mathrm{d} w}{\left(1-u_{1} w\right)(-w)^{q+1}} .
$$

Deforming the $v_{1}$-contour to $\infty$, we pick up the residue at $v_{1}=-u_{1}$ which gives

$$
\frac{-1}{(2 \pi \mathrm{i})^{2}} \oint \oint_{\left|u_{1} w\right|<1} \frac{\phi_{-}\left(u_{1}\right) \phi_{+}\left(w^{-1}\right)}{\phi_{+}\left(u_{1}\right) \phi_{-}\left(w^{-1}\right)} \frac{\mathrm{d} u_{1} \mathrm{~d} w}{\left(1-u_{1} w\right)\left(-u_{1}\right)^{p+1}(-w)^{q+1}},
$$

and this is exactly $\left(K_{11}\right)_{p q}-\delta_{p q}$. The proof of the formula for $K_{11}$ is complete.

In order to prove the formula for $K_{12}$, we need to show that $\left(1+A^{t} B\right) K_{12}=A^{t}$ or $A^{t} B K_{12}=A^{t}-K_{12}$. The computation of $A^{t} B K_{12}$ literally follows the above arguments for $K_{11}$ and leads to the sum of two terms:

$$
\begin{aligned}
\frac{1}{(2 \pi \mathrm{i})^{2}} \oint \oint_{\left|v_{1} w\right|<1} & \frac{\phi_{+}\left(w^{-1}\right)}{\phi_{+}\left(-v_{1}\right)} \frac{w^{q} \mathrm{~d} v_{1} \mathrm{~d} w}{\left(1+v_{1} w\right) v_{1}^{p+1}} \\
+ & \frac{1}{(2 \pi \mathrm{i})^{2}} \oint \oint_{\left|u_{1} w\right|<1} \frac{\phi_{-}\left(u_{1}\right) \phi_{+}\left(w^{-1}\right)}{\phi_{+}\left(u_{1}\right) \phi_{-}\left(w^{-1}\right)} \frac{w^{q} \mathrm{~d} u_{1} \mathrm{~d} w}{\left(1-u_{1} w\right)\left(-u_{1}\right)^{p+1}} .
\end{aligned}
$$

The first term is immediately seen to be equal to $A_{q p}$ and the second one is exactly $-\left(K_{12}\right)_{p q}$. 


\section{Schur functions and Schur measures}

We refer the reader to $[\mathrm{M}, \mathrm{S}]$ for general information on partitions and symmetric functions.

Let $\Lambda$ be the algebra of symmetric functions. It can be viewed as the algebra of polynomials in countably many indeterminates $\Lambda=\mathbb{C}\left[h_{1}, h_{2}, \ldots\right]$ where the indeterminates $h_{k}$ are the complete homogeneous symmetric functions of degree $k$. We also agree that $h_{0}=1$ and $h_{-k}=0$ for $k<0$.

The Schur symmetric functions $s_{\lambda}$ are parameterized by partitions $\lambda$ and are expressed through $h_{k} \mathrm{~s}$ by the Jacobi-Trudi formula

$$
s_{\lambda}=\operatorname{det}\left[h_{\lambda_{i}-i+j}\right]_{i, j=1}^{N}
$$

where $N$ is any number greater or equal to the number of nonzero parts of $\lambda$. The Schur functions form a linear basis in $\Lambda$.

An algebra homomorphism $\pi: \Lambda \rightarrow \mathbb{C}$ is uniquely determined by its values on $h_{k}$ s or by generating series of these values

$$
H^{\pi}(z)=\sum_{z=0}^{\infty} \pi\left(h_{n}\right) z^{n}
$$

Recall that a sequence $\left\{a_{n}\right\}_{n=0}^{\infty}$ is called totally positive if all minors of the matrix $\left[a_{i-j}\right]_{i, j \geqslant 0}$ are nonnegative. Here, all $a_{-k}$ for $k>0$ are assumed to be equal to zero. We will only consider totally positive sequences with $a_{0}=1$; clearly, multiplication of all members of a sequence by the same positive number does not affect total positivity.

The following statement was independently proved by Aissen-Edrei-SchoenbergWhitney in 1951 [AESW, E] and by Thoma in 1964 [T]. An excellent exposition of deep relations of this result to representation theory of the infinite symmetric group can be found in Kerov's book [K].

Theorem 2. A sequence $\left\{a_{n}\right\}_{n=0}^{\infty}, a_{0}=1$, is totally positive if and only if its generating series has the form

$$
\sum_{n=0}^{\infty} a_{n} z^{n}=\mathrm{e}^{\gamma z} \frac{\prod_{i \geqslant 1}\left(1+\beta_{i} z\right)}{\prod_{i \geqslant 1}\left(1-\alpha_{i} z\right)}=: F(\alpha, \beta, \gamma)
$$

for certain nonnegative parameters $\left\{\alpha_{i}\right\},\left\{\beta_{i}\right\}$ and $\gamma$ such that $\sum_{i}\left(\alpha_{i}+\beta_{i}\right)<\infty$.

Equivalently, an algebra homomorphism $\pi: \Lambda \rightarrow \mathbb{C}$ takes nonnegative values on all Schur functions if and only if the sequence $\left\{\pi\left(h_{n}\right)\right\}_{n \geqslant 0}$ is totally positive, that is, $H^{\pi}(z)=F(\alpha, \beta, \gamma)$ for a suitable choice of parameters $(\alpha, \beta, \gamma)$.

We will call a specialization $\pi: \Lambda \rightarrow \mathbb{C}$ positive if $\pi\left(s_{\lambda}\right) \geqslant 0$ for all partitions $\lambda$. Thus, the theorem above may be viewed as a classification of all positive specializations of the algebra of symmetric functions.

Let us now consider specializations $\pi_{+}$and $\pi_{-}$of $\Lambda$ such that

$$
H^{\pi_{+}}(z)=\phi_{+}(z), \quad H^{\pi_{-}}(z)=\phi_{-}\left(z^{-1}\right)
$$

for the holomorphic functions $\phi^{ \pm}$of the previous section, and set $s_{\lambda}^{ \pm}:=\pi_{ \pm}\left(s_{\lambda}\right)$.

Following Okounkov [O] assign to any partition $\lambda$, the following (generally speaking, complex) weight:

$$
P\{\lambda\}=\frac{s_{\lambda}^{+} s_{\lambda}^{-}}{Z}, \quad Z=\exp \sum_{k \geqslant 1} k\left(\ln \phi_{+}(z)\right)_{k}\left(\ln \phi_{-}(z)\right)_{k} .
$$


One can show that $\sum_{\lambda} P\{\lambda\}$ is an absolutely convergent series with sum equal to 1 . The distribution $P$ is called the Schur measure.

Theorem 1 proved in the previous section yields a new proof of the determinantal formula for the correlation functions of the Schur measure.

The following statement was proved in [O]; other proofs can be found in $[\mathrm{J}, \mathrm{R}]$.

For any partition $\lambda$, denote by $\mathcal{L}(\lambda)$ the infinite subset $\left\{\lambda_{i}-i\right\}_{i=1}^{\infty}$ of $\mathbb{Z}$.

Theorem 3. For any $x_{1}, \ldots, x_{n} \in \mathbb{Z}$

$$
\sum_{\lambda: \mathcal{L}(\lambda) \supset\left\{x_{1}, \ldots, x_{n}\right\}} P\{\lambda\}=\operatorname{det}\left[\mathcal{K}\left(x_{i}, x_{j}\right)\right]_{i, j=1}^{n}
$$

where

$$
\mathcal{K}(x, y)=\frac{1}{(2 \pi \mathrm{i})^{2}} \oint \oint_{|z w|<1} \frac{\phi_{-}(z) \phi_{+}\left(w^{-1}\right)}{\phi_{+}(z) \phi_{-}\left(w^{-1}\right)} \frac{z^{p} w^{q} \mathrm{~d} z \mathrm{~d} w}{1-z w} .
$$

Proof. We will use the material of the appendix in [BOO]. Let $\lambda$ be a partition and $\left(p_{1}, \ldots, p_{d} \mid q_{1}, \ldots, q_{d}\right)$ be its Frobenius coordinates ${ }^{1}$. Using the well-known Giambelli formula $s_{\lambda}=\operatorname{det}\left[s_{\left(p_{i} \mid q_{j}\right)}\right]_{i, j=1}^{d}$, see, e.g., [M, example I.3.9], we obtain

$$
P\{\lambda\}=\frac{s_{\lambda}^{+} s_{\lambda}^{-}}{Z}=Z^{-1} \operatorname{det}\left[s_{\left(p_{i} \mid q_{j}\right)}^{+}\right]_{i, j=1}^{d} \operatorname{det}\left[s_{\left(p_{i} \mid q_{j}\right)}^{-}\right]_{i, j=1}^{d} .
$$

Comparing the following formula for the generating series of the hook Schur functions

$$
1+(u+v) \sum_{p, q \geqslant 0} s_{(p \mid q)} u^{p} v^{q}=\frac{H(u)}{H(-v)}, \quad H(z)=\sum_{n=0}^{\infty} h_{n} z^{n},
$$

see, e.g., [M, example I.3.14], with the definition of matrices $A$ and $B$ in the previous section we see that

$$
s_{(p \mid q)}^{+}=A_{p q}, \quad s_{(p \mid q)}^{-}=B_{p q}, \quad p, q \geqslant 0 .
$$

Thus,

$$
P\{\lambda\}=Z^{-1} \cdot \operatorname{det}\left[A_{p_{i} q_{j}}\right]_{i, j=1}^{d} \operatorname{det}\left[B_{p_{i} q_{j}}\right]_{i, j=1}^{d}
$$

is, up to a constant, the value of the symmetric minor of the matrix $L$ from the previous section with $2 d$ rows and $2 d$ columns marked by $\left(q_{1}, \ldots, q_{d} \mid p_{1}, \ldots, p_{d}\right)$. This means that the Schur measure interpreted through the Frobenius coordinates of partitions defines a determinantal point process on $\mathbb{Z}_{+} \sqcup \mathbb{Z}_{+}$, and its correlation kernel is given by $K=L(1+L)^{-1}$. (The operator $1+L$ is invertible because $\operatorname{det}(1+L)=Z \neq 0$.)

Finally, to pass from Frobenius coordinates $\left(p_{1}, \ldots, p_{d} \mid q_{1}, \ldots, q_{d}\right) \subset \mathbb{Z}_{+} \sqcup \mathbb{Z}_{+}$to $\mathcal{L}(\lambda) \subset \mathbb{Z}$ we can use the complementation principle, see [appendix A.3, BOO], thanks to the Frobenius lemma

$$
\mathcal{L}(\lambda)=\left\{p_{1}, \ldots, p_{d}\right\} \sqcup\left(\{-1,-2,-3, \ldots\} \backslash\left\{-q_{1}-1, \ldots,-q_{d}-1\right\}\right),
$$

see, e.g., [M, I.1(1.7)]. It is readily seen that the operation $\triangle$ of [appendix A.3, BOO] transforms the kernel $K$ of theorem 1 to the kernel $\mathcal{K}$ of theorem 3 up to a conjugation by a diagonal matrix of plus-minus $1 \mathrm{~s}$. Since such conjugation does not change the determinants $\operatorname{det}\left[\mathcal{K}\left(x_{i}, x_{j}\right)\right]_{i, j=1}^{n}$, the proof is complete.

1 Frobenius coordinate $p_{i}$ measures the number of boxes in the $i$ th row of the corresponding Young diagram to the right of the diagonal. Similarly, $q_{j}$ is the number of boxes in the $j$ th column below the diagonal. Formally, $p_{i}=\lambda_{i}-i, q_{i}=\lambda_{i}^{\prime}-i, i=1, \ldots, d$, where $d$ is the length of the diagonal of the Young diagram corresponding to $\lambda$. 


\section{Total positivity and Widom's conjecture}

In this section, we assume that the functions $\phi_{+}(z)$ and $\phi_{-}\left(z^{-1}\right)$ are generating functions of totally positive sequences, that is,

$$
\phi_{+}(z)=\mathrm{e}^{\gamma^{+} z} \frac{\prod_{i \geqslant 1}\left(1+\beta_{i}^{+} z\right)}{\prod_{i \geqslant 1}\left(1-\alpha_{i}^{+} z\right)}, \quad \phi_{-}(z)=\mathrm{e}^{\gamma^{-} / z} \frac{\prod_{i \geqslant 1}\left(1+\beta_{i}^{-} / z\right)}{\prod_{i \geqslant 1}\left(1-\alpha_{i}^{-} / z\right)}
$$

for certain nonnegative parameters $\left\{\alpha_{i}^{ \pm}\right\},\left\{\beta_{i}^{ \pm}\right\}$and $\gamma^{ \pm}$such that $\sum_{i}\left(\alpha_{i}^{ \pm}+\beta_{i}^{ \pm}\right)<\infty$ and $\alpha_{i}^{ \pm}, \beta_{i}^{ \pm}<r^{-1}<1$ for all $i \geqslant 1$.

Let $P_{n}$ be the projection operator in $L^{2}\left(\mathbb{Z}_{+}\right)$which projects onto $n$ first basis vectors:

$$
P_{n}:\left(x_{0}, x_{1}, x_{2}, \ldots\right) \mapsto\left(x_{0}, x_{1}, \ldots, x_{n-1}, 0,0, \ldots\right) .
$$

Theorem 4. Under the above assumption, for any $n=1,2, \ldots$ the spectra of the operators $K_{11}^{(n)}=\left(1-P_{n}\right) K_{11}\left(1-P_{n}\right)$ and $K_{22}^{(n)}=\left(1-P_{n}\right) K_{22}\left(1-P_{n}\right)$ are real and lie between 0 and 1 .

Let us start with a lemma.

Lemma 5. Let $T_{n}$ be a sequence of trace class operators in a Hilbert space which converges to a (trace class) operator $T$ in trace norm. Assume that $S p\left(T_{n}\right) \subset S$ for a closed set $S \subset \mathbb{C}$ and all large enough $n$. Then, $S p(T) \subset S \cup\{0\}$.

Proof. By virtue of (proposition A.11, [BOO]), trace norm convergence implies the convergence of entire functions in $z$

$$
\operatorname{det}\left(1+z T_{n}\right) \rightarrow \operatorname{det}(1+z T)
$$

uniformly on compact subsets of $\mathbb{C}$. Therefore, any zero of $\operatorname{det}(1+z T)$ is a limit point of zeros of $\operatorname{det}\left(1+z K_{n}\right)$.

Proof of theorem 4. Let us first show that $S p\left(K_{11}\right)$ and $S p\left(K_{22}\right)$ lie inside the interval $[0,1)$.

Since the specializations $\pi_{ \pm}$associated with $\phi_{ \pm}$are positive, the matrices $A$ and $B$ are totally positive. Indeed, by Giambelli's formula their minors are values of $\pi_{ \pm}$on appropriate Schur functions. Furthermore, recall that $A_{p q}$ and $B_{p q}$ decay faster than const $\cdot x^{-p-q}$ for any $1<x<r$ as $p+q \rightarrow \infty$. This means that the matrix $A^{t} B$ is also totally positive and its matrix elements satisfy similar estimates. Consequently, $A^{t} B$ is a trace class operator, and $\operatorname{det}\left(1+A^{t} B\right)-1$ is equal to the sum of all symmetric minors of $A^{t} B$, which is nonnegative. Thus, the operator $1+A^{t} B$ is invertible.

The decay of the matrix elements of $A^{t} B$ shows that $P_{n} A^{t} B P_{n}$ converges to $A^{t} B$ in trace norm as $n \rightarrow \infty$ (indeed, the trace norm of a matrix does not exceed the sum of the absolute values of the matrix elements). It is well known that the eigenvalues of a totally positive matrix are nonnegative, see, e.g., [An, corollary 6.6]. Hence, $S p\left(P_{n} A^{t} B P_{n}\right) \subset \mathbb{R}_{\geqslant 0}$, and by lemma 5, $S p\left(A^{t} B\right) \subset \mathbb{R}_{\geqslant 0}$. Therefore, $\operatorname{Sp}\left(K_{11}\right)=A^{t} B\left(1+A^{t} B\right)^{-1} \subset[0,1)$. The argument for $K_{22}$ is very similar.

To extend the argument to $K_{11}^{(n)}, K_{22}^{(n)}$, we need a linear algebraic lemma.

Lemma 6. Let $C$ be an $(m+n) \times(m+n)$ matrix such that $1+C$ is invertible and let $D$ be the $n \times n$ lower right corner of $C(1+C)^{-1}$. Then,

$$
\operatorname{det}(1-D)=\sum_{X \subset\{1, \ldots, m\}} \frac{C\left(\begin{array}{l}
X \\
X
\end{array}\right)}{\operatorname{det}(1+C)}
$$


(the sum above includes $X=\varnothing$ and $\left.C\left(\begin{array}{l}\varnothing \\ \varnothing\end{array}\right)=1\right)$. Assume further that $\operatorname{det}(1-D) \neq 0$ and set $E=D(1-D)^{-1}$. Then any minor of $E$ is, up to a constant, a sum of certain minors of the initial matrix $C$ :

$$
\frac{E\left(\begin{array}{l}
X \\
Y
\end{array}\right)}{\operatorname{det}(1+E)}=\sum_{Z \subset\{1, \ldots, m\}} \frac{C\left(\begin{array}{l}
Z \sqcup X \\
Z \sqcup Y
\end{array}\right)}{\operatorname{det}(1+C)} .
$$

Proof. The first identity follows from the formula for the minors of the inverse

$$
\operatorname{det}(1-D)=\frac{\operatorname{det} G}{\operatorname{det}(1+C)}=\sum_{X \subset\{1, \ldots, m\}} \frac{C\left(\begin{array}{l}
X \\
X
\end{array}\right)}{\operatorname{det}(1+C)},
$$

where $G$ is the $m \times m$ upper-left corner of $1+C$.

There are two ways to compute minors of $1-D$, one of them using $1+E$ and the other using $1+C$. This yields

$$
(1+E)\left(\begin{array}{l}
X \\
Y
\end{array}\right)=\frac{\operatorname{det}(1+E)}{\operatorname{det}(1+C)}(1+C)\left(\begin{array}{c}
1, \ldots, m, X \\
1, \ldots, m, Y
\end{array}\right)
$$

In the case when $X$ and $Y$ do not intersect, the last identity implies the second identity of the lemma. When $X$ and $Y$ intersect, expansion of the left-hand side and the right-hand side into a linear combination of minors of $E$ and $C$, respectively, yields the second identity of the lemma by induction.

Now we can apply this statement to the totally positive matrices $C=P_{m} A^{t} B P_{m}, m>n$. Then, clearly, $\operatorname{det}(1-D)>0$, and $E=D(1-D)^{-1}$ with

$$
D=\left(1-P_{n}\right) \frac{P_{m} A^{t} B P_{m}}{1+P_{m} A^{t} B P_{m}}\left(1-P_{n}\right),
$$

is totally positive. Since eigenvalues of totally positive matrices are nonnegative, we obtain $S p(D) \subset[0,1)$. As was mentioned earlier, $P_{m} A^{t} B P_{m} \rightarrow A^{t} B$ in trace norm as $m \rightarrow \infty$. Hence, $D \rightarrow K_{11}^{(n)}$ in trace norm as $m \rightarrow \infty$, and lemma 5 implies that $\operatorname{Sp}\left(K_{11}^{(n)}\right) \subset[0,1]$. The case of $K_{22}^{(n)}$ is handled similarly.

Note now that by expanding $(1-z w)^{-1}=1+z w+(z w)^{2}+\cdots$ in the integral representation for $K_{11}$, we obtain

$$
\left(K_{11}\right)_{p q}=\delta_{p q}-(-1)^{p+q} \sum_{k \geqslant 0}\left(\frac{\phi_{-}}{\phi_{+}}\right)_{p-k}\left(\frac{\phi_{+}}{\phi_{-}}\right)_{-q+k}=(-1)^{p+q} \sum_{k \geqslant 1}\left(\frac{\phi_{-}}{\phi_{+}}\right)_{p+k}\left(\frac{\phi_{+}}{\phi_{-}}\right)_{-q-k} .
$$

Widom in [W] conjectured that the eigenvalues of the operator in $L^{2}(n, n+1, \ldots)$ with $(p, q)$-matrix element

$$
\sum_{k \geqslant 1}\left(\frac{\phi_{-}}{\phi_{+}}\right)_{p+k}\left(\frac{\phi_{+}}{\phi_{-}}\right)_{-q-k}
$$

lie between 0 and 1 for the specific choice of

$$
\phi_{+}(z)=\prod_{i \geqslant 1}\left(1+r_{i} z\right), \quad \phi_{-}(z)=\prod_{i \geqslant 1}\left(1-s_{i} z^{-1}\right)^{-1}
$$

with nonnegative $r_{i}$ and $s_{i}$ only finitely many of which are nonzero; $r_{i}, s_{i}<1$. Clearly, this statement is an immediate corollary of theorem 4. 


\section{Acknowledgment}

One of the authors (AB) was partially supported by the NSF grant DMS-0402047.

\section{References}

[AESW] Aissen M, Edrei A, Schoenberg I J and Whitney A 1951 On the generating functions of totally positive sequences Proc. Natl Acad. Sci. USA 37 303-7

[An] Ando T 1987 Totally positive matrices Linear Algebra Appl. 90 165-219

[BDR] Baik J, Deift P and Rains E 2001 A Fredholm determinant identity and the convergence of moments for random Young tableaux Commun. Math. Phys. 223 627-72 (Preprint math/0012117)

[BOO] Borodin A, Okounkov A and Olshanski G 2000 Asymptotics of Plancherel measures for symmetric groups J. Am. Math. Soc. 13 491-515 (Preprint math/9905032)

[E] Edrei A 1952 On the generating functions of totally positive sequences: II J. Anal. Math. 2 104-9

[J] Johansson K 2001 Random growth and random matrices European Congress of Mathematics (Barcelona) vol I (Basle: Birkhäuser)

[K] Kerov S V 2003 Asymptotic Representation Theory of the Symmetric Group and its Applications in Analysis (Translations of Mathematical Monographs vol 219) (Providence, RI: American Mathematical Society)

[M] Macdonald I G 1995 Symmetric Functions and Hall Polynomials 2nd edn (Oxford: Oxford University Press)

[O] Okounkov A 2001 Infinite wedge and measures on partitions Sel. Math. 7 1-25 (Preprint math/9907127)

[R] Rains E M 2000 Correlation functions for symmetrized increasing subsequences Preprint math/0006097

[S] Stanley R P 1999 Enumerative Combinatorics vol 2 (Cambridge: Cambridge University Press)

[T] Thoma E 1964 Die unzerlegbaren, positive-definiten klassenfunktionen der abzählbar unendlichen, symmetrischen gruppe Math. Z. 85 40-61

[W] Widom H 2002 On convergence of moments for random Young tableaux and a random growth model Int. Math. Res. Not. 9 455-64 